\title{
Novas Ferramentas para o Tratamento Percutâneo de Defeitos Congênitos
}

\author{
Ver artigo relacionado \\ na página 81
}

\author{
Carlos Zabal ${ }^{1}$
}

A oclusão da comunicação interatrial transcateter foi descrita pela primeira vez por King e Mills, em 1976. ${ }^{1}$ Alguns casos iniciais tiveram êxito, porém o alto perfil do sistema limitava seu uso exclusivamente a pacientes idosos. Em 1983, Rashkind ${ }^{2}$ descreveu o uso de um dispositivo com disco único, que foi o precursor de dispositivos posteriores com sistema de duplo disco, dando início à corrida tecnológica para o desenvolvimento de dispositivos de oclusão. Nos últimos vinte anos, essa corrida tecnológica revelou diferentes dispositivos, embora nenhum deles seja o ideal para todos os tipos, formas e tamanhos de defeitos. Até o momento, o dispositivo mais próximo de ser considerado ideal é o oclusor septal Amplatzer (Amplatzer Septal Occluder; AGA Medical Corporation, Golden Valley, Estados Unidos), que apresenta as seguintes vantagens: sistema de colocação e liberação muito fácil e simples, que permite recolocação, reposicionamento ou até mesmo extração sem que o dispositivo sofra danos, além de poder ocluir grandes defeitos, em alguns casos selecionados com até mais de $40 \mathrm{~mm}$. Recentemente, foi disponibilizado no mercado o dispositivo Figulla (Occlutech), tema do artigo de Pedra et al. ${ }^{3}$ publicado nesta edição da Revista Brasileira de Cardiologia Invasiva. Segundo esses autores, o dispositivo é muito semelhante à prótese Amplatzer, de forma que as equipes com ampla experiência no uso desse dispositivo não terão dificuldade para utilizar a prótese Figulla.

Ainda segundo Pedra et al., ${ }^{3}$ a versatilidade dessas próteses ampliou o espectro de indicação para oclusão percutânea dos defeitos septais. Pode-se dizer que, na atualidade, $90 \%$ das comunicações interatriais tipo ostium secundum e cerca de $80 \%$ de todos os defeitos do septo atrial podem ser tratados por via percutânea. ${ }^{4}$ Acredita-se, portanto, que o tratamento percutâneo de adultos com mais de 40 anos de idade com comunicação interatrial é muito útil, já que esse tipo de abordagem apresenta menos complicações que o tratamento cirúrgico nesse grupo de pacientes. ${ }^{5}$

Obviamente, ainda existem problemas, como relatam Pedra et al. ${ }^{3}$ em seu artigo. Alguns casos requerem técnicas de colocação especiais, principalmente os defeitos grandes com remanescentes septais pequenos ou deficientes. A preocupação atual com esse tipo de dispositivo tem sido nos raros casos descritos de erosão da raiz aórtica com fístulas atriais ou do teto dos átrios com consequente tamponamento cardíaco e possível morte. ${ }^{6}$ Esse fato está relacionado ao uso de dispositivos com diâmetro muito maior que o tamanho do defeito, razão pela qual se recomenda não sobredimensionar os dispositivos de forma excessiva. É provável que o dispositivo Figulla tenha um comportamento diferente nesse sentido, pela sua maior maleabilidade, porém apenas o tempo poderá confirmar essa hipótese. Em nossa experiência com mais de 600 casos tratados com a prótese Amplatzer, ainda não tivemos casos com essa grave complicação.

Concluindo, graças à atual corrida tecnológica, já temos disponíveis no mercado vários tipos de prótese para o tratamento transcateter dos defeitos do septo atrial, o que nos ajudou a revolucionar e a massificar o tratamento percutâneo dos defeitos congênitos.

\section{CONFLITO DE INTERESSES}

O autor é proctor e consultor da AGA Medical Corporation.

\section{REFERÊNCIAS}

1. King TD, Thompson SL, Steiner C, Mills NL. Secundum atrial septal defect: nonoperative closure during cardiac catheterization. JAMA. 1976;235(23):2506-9.

2. Rashkind WJ. Transcatheter treatment of congenital heart diseases. Circulation. 1983;67(4):711-6.

\footnotetext{
1 Instituto Nacional de Cardiologia Ignacio Chávez - México, D.F., México.

Correspondência: Carlos Zabal. Juan Badiano 1 - Col. Sección XVI - Tlalpan - Mexico, D.F., Mexico - 14080

E-mail: zabal@cardiologia.org.mx

Recebido em: 12/3/2010 • Aceito em: 13/3/2010
} 
3. Pedra CAC, Pedra SRF, Costa RN, Braga SLN, Esteves CA, Fontes VF. Experiência inicial no fechamento percutâneo da comunicação interatrial tipo ostium secundum com a prótese Figulla. Rev Bras Cardiol Invasiva. 2010;18(1):81-8.

4. Butera G, Romagnoli E, Carminati M, Chessa M, Piazza L, Negura D, et al. Treatment of isolated secundum atrial septal defects: impact of age and defect morphology in 1,013 consecutive patients. Am Heart J. 2008;156(4):706-12.

5. Rosas M, Zabal C, García-Montes J, Buendía A, Webb G,
Attie F. Transcatheter versus surgical closure of secundum atrial septal defect in adults: impact of age at intervention. A concurrent matched comparative study. Congenital Heart Dis. 2007;2(3):148-55.

6. Amin Z, Hijazi ZM, Bass JL, Cheatham JP, Hellebrand WE, Kleinman CS. Erosion of Amplatzer septal occluder device after closure of secundum atrial septal defects: review of registry of complications and recommendations to minimize future risk. Catheter Cardiovasc Interv. 2004;63(4):496-502. 